\title{
ALICE probes of local parity violation with charge dependent azimuthal correlations in $\mathrm{Pb}-\mathrm{Pb}$ collisions
}

Ilya Selyuzhenkov* for the ALICE Collaboration

Research Division and ExtreMe Matter Institute EMMI,

GSI Helmholtzzentrum für Schwerionenforschung, Darmstadt, Germany E-mail:

ilya.selyuzhenkovegmail.com

\begin{abstract}
We report on the measurement of two and three particle azimuthal correlations in $\mathrm{Pb}-\mathrm{Pb}$ collisions at $\sqrt{s_{N N}}=2.76 \mathrm{TeV}$ recorded with ALICE at the LHC. While two particle azimuthal correlations mainly provide an important information on possible background correlations, the three particle correlator probes the charge separation of hadrons with respect to the collision reaction plane which is expected for local parity violation in strong interactions. The two and three particle correlations are presented as a function of collision centrality and differentially vs. pseudorapidity and transverse momentum, and provide strong constraints on the possible mechanism of background (parity conserving) and signal (parity odd) effects in heavy-ion collisions.
\end{abstract}

The Seventh Workshop on Particle Correlations and Femtoscopy

September 20 - 242011

University of Tokyo, Japan

\footnotetext{
*Speaker.
} 


\section{Introduction}

An extreme magnetic field created during a non-central relativistic heavy-ion collision may spontaneously excite instantons and sphalerons from the QCD vacuum which violates parity symmetry of the strong interactions. It is argued by Kharzeev et al. [1] that this may result in the experimentally observable separation of charges along the magnetic field. Thus, a measurement of charge separation with respect to the reaction plane for hadrons produced in heavy-ion collisions provides a unique way to experimentally probe the phenomena of local parity violation in strong interactions which has been discussed for many years [1,2,3]. The measurement of the charge dependent correlations by the STAR Collaboration [4] revealed a signal which is qualitatively consistent with the expectation for the charge separation from local parity violation. This triggered an intensive discussion about possible interpretation of the correlations $[5,6,7,8]$. In these proceedings I report on the charge dependent azimuthal correlations measured at mid-rapidity for $\mathrm{Pb}-\mathrm{Pb}$ collisions at $\sqrt{s_{N N}}=2.76 \mathrm{TeV}$ with the ALICE detector $[9,10]$ at the LHC.

\section{Observables and experimental data}

An experimental observable which is sensitive to effects from local parity violation in strong interactions was proposed in [11]:

$$
\left\langle\cos \left(\phi_{\alpha}+\phi_{\beta}-2 \Psi_{R P}\right)\right\rangle=\left\langle\cos \Delta \phi_{\alpha} \cos \Delta \phi_{\beta}\right\rangle-\left\langle\sin \Delta \phi_{\alpha} \sin \Delta \phi_{\beta}\right\rangle .
$$

Here $\phi_{\alpha, \beta}$ are the positive or negative charged particle azimuthal angles, $\Delta \phi=\phi-\Psi_{R P}$, and $\Psi_{R P}$ is the reaction plane angle. Experimentally the reaction plane is estimated from the azimuthal asymmetry of the produced particles and (2.1) become a three particle correlator. In addition to (2.1), the measurement of the two-particle correlator

$$
\left\langle\cos \left(\phi_{\alpha}-\phi_{\beta}\right\rangle=\left\langle\cos \Delta \phi_{\alpha} \cos \Delta \phi_{\beta}\right\rangle+\left\langle\sin \Delta \phi_{\alpha} \sin \Delta \phi_{\beta}\right\rangle\right.
$$

provides an experimental constraint on models of possible parity-even background contributions to (2.1). Together, the two (2.2) and three (2.1) particle correlators supply information about inplane, $\left\langle\cos \Delta \phi_{\alpha} \cos \Delta \phi_{\beta}\right\rangle$, and out-of-plane, $\left\langle\sin \Delta \phi_{\alpha} \sin \Delta \phi_{\beta}\right\rangle$, charge correlations.

The ALICE detector description and performance is given in [9]. For this study the Time Projection Chamber (TPC) [12], two forward scintillator arrays (VZERO) with pseudorapidity coverage $-3.7<\eta<-1.7$ and $2.8<\eta<5.1$, and two neutron Zero Degree Calorimeters (ZDC) [9] located at about $114 \mathrm{~m}$ on both sides of the interaction point are used. The TPC, VZERO detectors, and neutron ZDCs are used to estimate the orientation of the reaction plane. We used a sample of about 13 million minimum-bias $\mathrm{Pb}-\mathrm{Pb}$ collisions at $\sqrt{s_{N N}}=2.76 \mathrm{TeV}$ recorded by the ALICE detector in 2010. The standard ALICE event selection criteria with the collision centrality estimated from VZERO detectors [13] and a collision vertex cut of $7 \mathrm{~cm}$ along the beam axis were applied. Only charged particles with pseudorapidity $|\eta|<0.8$ and transverse momentum $p_{\mathrm{T}}>0.2 \mathrm{GeV} / \mathrm{c}$ are used for the measurements.

The systematic uncertainties were evaluated based on the comparison between results obtained with opposite magnetic field polarities and variation of the results with the cut on the collision 
vertex along the beam direction within $\pm 10 \mathrm{~cm}$. The bias in the collision centrality determination is estimated from the difference between results with the centrality estimated from VZEROs, TPC and Silicon Pixel Detector (SPD). Contamination from charged tracks which did not originate from the collision vertex was estimated by varying the cut on the distance of closest approach in the transverse plane between the track and reconstructed collision vertex within $4 \mathrm{~cm}$. Effects due to non-uniform acceptance of the TPC are found to be below $2 \%$ and are corrected for in the final result. The total systematic uncertainty is obtained by adding uncertainties from all the sources in quadrature.

\section{Results}

Figure 1(a) shows the 3-particle correlator (2.1) as a function of centrality calculated from four different analyses: 3 particle cumulants [14] and a set of three independent measurements with the

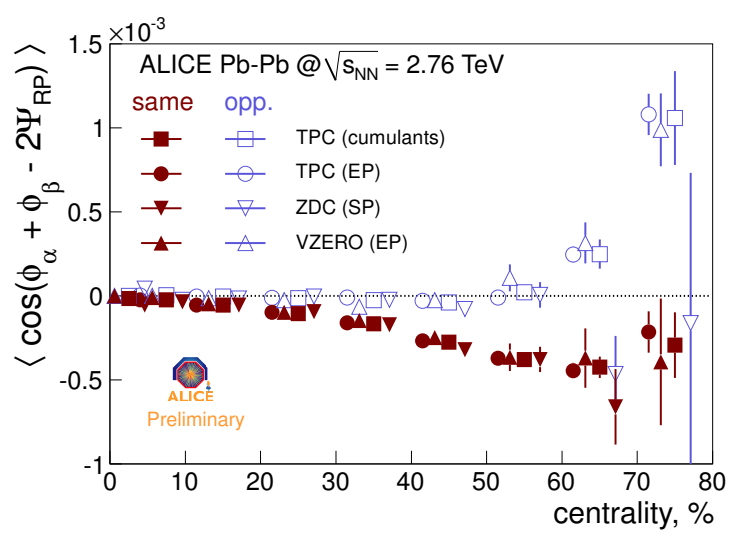

(a)

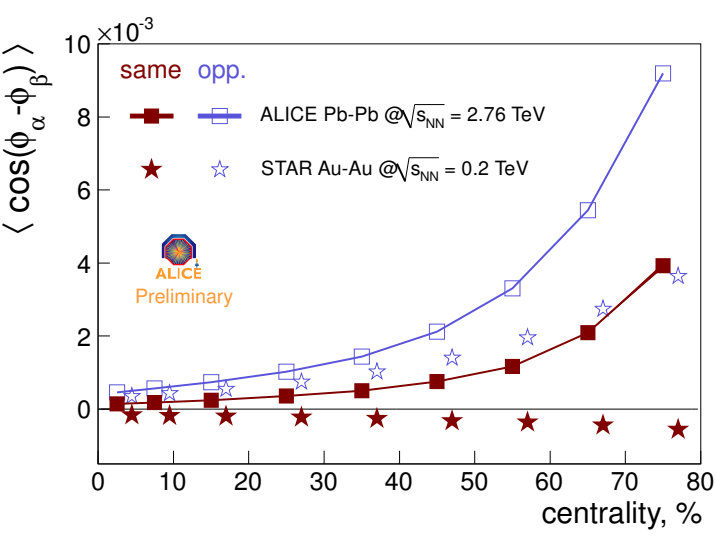

(b)

Figure 1: (Color online) (a) Centrality dependence of the 3-particle correlator measured with the reaction plane estimated from TPC, ZDCs and the VZEROs detectors. (b) Centrality dependence of the 2-particle correlations compared with the STAR data [4].

reaction plane estimated from different ALICE detector subsystems (TPC, ZDCs and VZEROs) using the scalar product (SP) and the event plane (EP) measurement techniques [15]. Agreement between four methods indicate that the measured three particle correlations represent two charged particle correlation with respect to the reaction plane. Small variations between methods were used to evaluate the total systematic uncertainty. The results for positive-positive and negative-negative pairs were combined into the same charge correlations. The observed difference in Fig. 1(a) between the same and opposite charge correlations may in part originates from effects of local parity violation in the strong interaction. However, a number of other charge dependent effects which preserve parity symmetry can also contribute. To constrain the possible backgrounds experimentally, in Fig. 1(b) we present the centrality dependence of the 2-particle correlator (2.2). The ALICE results for the 2-particle correlations differ from those reported by the STAR Collaboration [4].

Figure 2(a) presents the 3-particle correlator (2.1) measured by the ALICE Collaboration compared to the RHIC data [4] and correlations expected for the same charge pairs from effects of local parity violation at LHC energies [16]. Statistical (systematic) uncertainties in Fig. 2 are shown by 


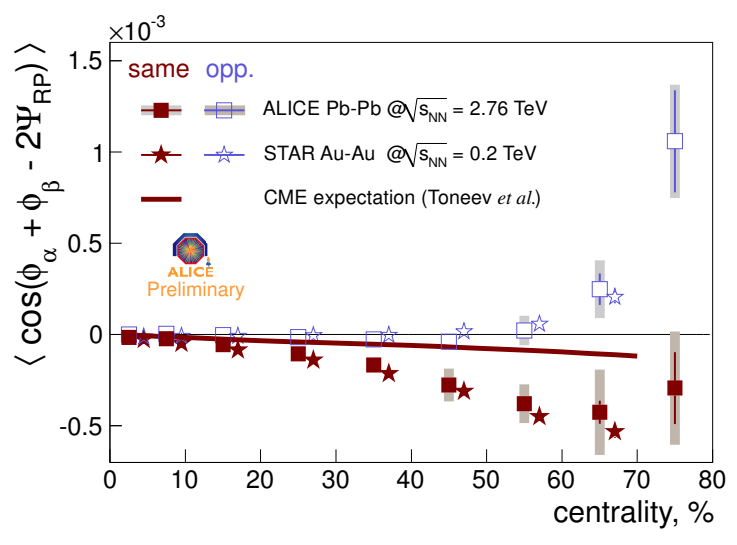

(a)

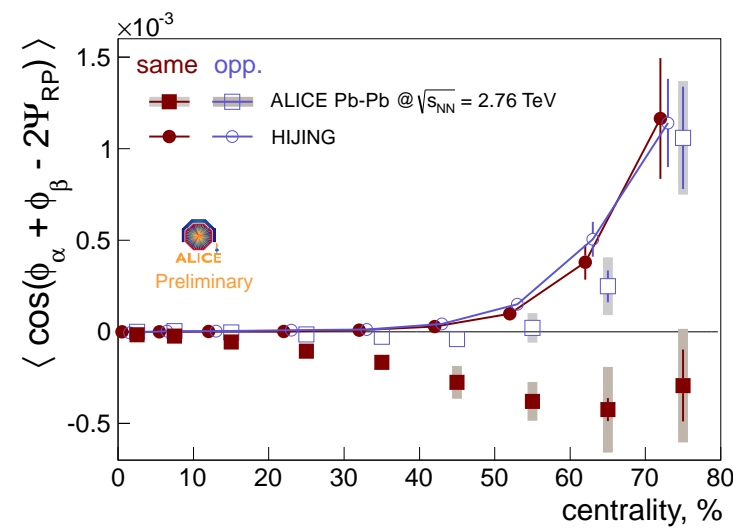

(b)

Figure 2: (color online) 3-particle correlator vs. centrality: (a) compared to STAR data [4] and theoretical predictions which incorporates effects of local parity violation at LHC energies [16], (b) expectations from HIJING [19] Monte-Carlo.

the error bars (shaded area) respectively. In addition to the sources listed in Sec. 2, the dominant contribution to the systematic error comes from the uncertainty in the measurement of the elliptic flow, $v_{2}$, which is used to estimate the event plane resolution. For corrections we used an average of the elliptic flow measured from 2- and 4-particle cumulants [13] which has different sensitivity to the correlations unrelated to the reaction plane (non-flow) and flow fluctuations. Half of the difference between $\mathrm{v}_{2}$ measured with 2- and 4-particle cumulants was assigned as the systematic uncertainty.

Figure 2(a) reveals a remarkable similarity between ALICE and STAR measurements performed at the collision energies $\sqrt{s_{N N}}=0.2$ and $2.76 \mathrm{TeV}$. Both the ALICE and STAR results show a strong correlation between pairs of the same charge, and, in contrast a weak correlation among oppositely charged pairs. If the observed charge dependence would be due to only the local parity violation this behavior can be interpreted as suppression (quenching) by the medium of the opposite charge sign correlations [17]. Another possible explanation for the difference in the magnitude of the correlation for same and opposite charge sign pairs can be a baseline shift due to charge independent fluctuations of the directed flow [18]. Together with the ALICE and STAR experimental data, Fig. 2(a) shows predictions for the parity violating effects at LHC energies based on a model [16] which incorporates the time evolution of the magnetic field. The model could not predict the actual magnitude of the charge correlations, and in the calculations the measured correlations by the STAR Collaboration were scaled to the LHC energies. While such predictions underestimate the observed magnitude of the same sign correlations at the LHC by a factor of five, it was noticed in $[8,17]$ that the strength of charge correlations can be the same for RHIC and LHC energies.

Figure 2(b) shows comparison between the three particle correlator (2.1) measured by ALICE and charge correlations extracted from HIJING [19] Monte-Carlo simulations for $\mathrm{Pb}-\mathrm{Pb}$ collisions at $\sqrt{s_{N N}}=2.76 \mathrm{TeV}$. The correlations in the HIJING simulations show almost no charge dependence, and significantly increase for very peripheral collisions, which is due to correlations not 
related to the reaction plane (see for example discussion in [4]). Note that in [6] it is argued that effects of local charge conservation may be responsible for a significant part of the observed charge dependence of the correlator (2.1).

The differential dependencies of the charge correlations provides another way to compare various models which attempt to describe the observed charge separation. Figure 3 shows the

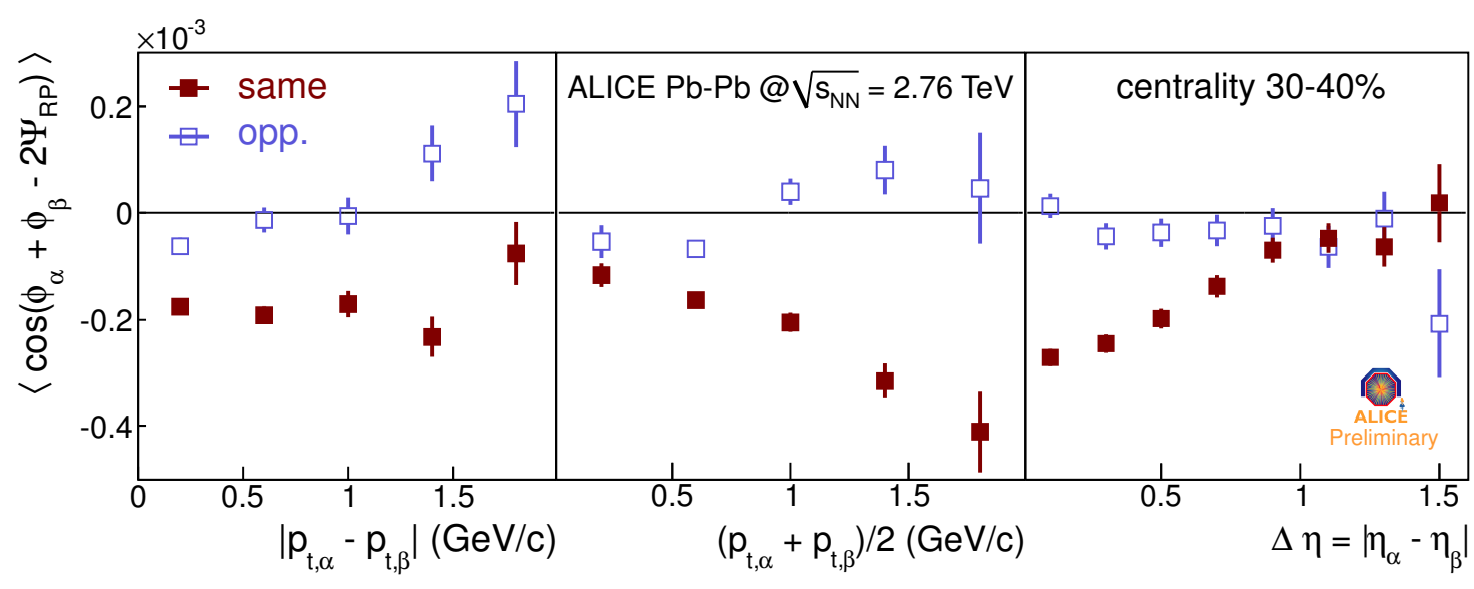

(a)

(b)

(c)

Figure 3: (Color online) 3-particle correlator as a function of the (a) difference and (b) mean pair transverse momentum, and (c) the pair separation in pseudorapidity.

3-particle correlator (2.1) as a function of the difference and mean pair transverse momentum, and the pair separation in pseudorapidity for the $30-40 \%$ centrality region. Compared to pairs of opposite charge, the same charge pairs show a strong increase in the magnitude with increasing mean transverse momentum of the pair (Fig. 3(b)), with a correlation width of about one unit in pseudorapidity (Fig. 3(c)).

\section{Summary}

Two and three particle charge dependent azimuthal correlations have been measured for $\mathrm{Pb}-\mathrm{Pb}$ collisions at $\sqrt{s_{N N}}=2.76 \mathrm{TeV}$ by ALICE at the LHC. A clear charge dependence of the correlations is observed. The three particle correlations are very similar to those reported by the STAR Collaboration for Au-Au collisions at $\sqrt{s_{N N}}=0.2 \mathrm{TeV}$. At the same time, the two particle correlations differ strongly from those observed at RHIC. Together, the two and three particle correlations measured at the LHC may provide strong experimental constraints on the possible mechanism of parity conserving and parity odd effects in heavy-ion collisions.

\section{Acknowledgment}

This work was supported by the Helmholtz Alliance Program of the Helmholtz Association, contract HA216/EMMI "Extremes of Density and Temperature: Cosmic Matter in the Laboratory". 


\section{References}

[1] D. Kharzeev, R. D. Pisarski and M. H. G. Tytgat, Phys. Rev. Lett. 81, 512 (1998).

D. Kharzeev and R. D. Pisarski, Phys. Rev. D61, 111901 (2000).

D. E. Kharzeev, L. D. McLerran and H. J. Warringa, Nucl. Phys. A 803, 227 (2008).

[2] T. D. Lee, Phys. Rev. D8, 1226 (1973).

T. D. Lee and G. C. Wick, Phys. Rev. D9, 2291 (1974).

[3] P. D. Morley and I. A. Schmidt, Z. Phys. C26, 627 (1985).

[4] J. Adams et al. [STAR Collaboration], Phys. Rev. Lett. 103, 251601 (2009).

J. Adams et al. [STAR Collaboration], Phys. Rev. C81, 54908 (2010).

[5] B. Muller and A. Schafer, Phys. Rev. C 82, 057902 (2010).

[6] S. Schlichting and S. Pratt, Phys. Rev. C 83, 014913 (2011).

S. Pratt, S. Schlichting and S. Gavin, Phys. Rev. C 84, 024909 (2011)

[7] A. Bzdak et al., Phys.Rev. C81, 031901 (2010).

A. Bzdak et al., Phys.Rev. C83, 014905 (2011).

[8] A. R. Zhitnitsky, arXiv:1201.2665 [hep-ph].

[9] K. Aamodt et al. [ALICE Collaboration], JINST 3, S08002 (2008).

[10] K. Aamodt et al. [ALICE Collaboration], J. Phys. G30, 1517 (2004).

K. Aamodt et al. [ALICE Collaboration], J. Phys. G32, 1295 (2006).

[11] S. A. Voloshin, Phys. Rev. C70, 057901 (2004).

[12] J. Alme et al. [ALICE Collaboration], Nucl. Instrum. Meth. A622, 316 (2010).

[13] K. Aamodt et al. [ALICE Collaboration], Phys. Rev. Lett. 105, 252302 (2010).

[14] A. Bilandzic, R. Snellings and S. Voloshin, Phys. Rev. C83, 044913 (2011).

[15] S. A. Voloshin, A. M. Poskanzer and R. Snellings, arXiv:0809.2949 [nucl-ex].

[16] V. D. Toneev and V. Voronyuk, arXiv:1012.1508 [nucl-th].

[17] D. Kharzeev, Phys. Lett. B633, 260 (2006).

D. Kharzeev, L. D. McLerran and H. J. Warringa, Nucl. Phys. A803, 227 (2008).

K. Fukushima, D. E. Kharzeev and H. J.Warringa, Phys. Rev. D78, 074033 (2008).

[18] D. Teaney and L. Yan, arXiv:1010.1876 [nucl-th].

[19] M. Gyulassy and X. N. Wang, Comput. Phys. Commun. 83, 307 (1994).

X. N. Wang and M. Gyulassy, Phys. Rev. D44, 3501 (1991). 